\title{
Quantum control of vibrational excitations in a heteronuclear diatomic molecule
}

\author{
SITANSH SHARMA, PURSHOTAM SHARMA and HARJINDER SINGH* \\ Center for Computational Natural Sciences and Bioinformatics, \\ International Institute of Information Technology, Hyderabad 500032 \\ e-mail: laltu@iiit.ac.in
}

MS received 28 April 2007; accepted 13 June 2007

\begin{abstract}
Optimal control theory is applied to obtain infrared laser pulses for selective vibrational excitation in a heteronuclear diatomic molecule. The problem of finding the optimized field is phrased as a maximization of a cost functional which depends on the laser field. A time dependent Gaussian factor is introduced in the field prior to evaluation of the cost functional for better field shape. Conjugate gradient method $^{21,24}$ is used for optimization of constructed cost functional. At each instant of time, the optimal electric field is calculated and used for the subsequent quantum dynamics, within the dipole approximation. The results are obtained using both Morse potential as well as potential energy obtained using ab initio calculations.
\end{abstract}

Keywords. Optimal control theory; conjugate gradient method; Morse potential; $a b$ initio calculations.

\section{Introduction}

Optical time-resolved techniques and frequencyresolved spectroscopic techniques have helped us to gain good knowledge of the electronic, vibrational and rotational dynamics in many molecules. ${ }^{1,2}$ The growing understanding of these quantum processes has opened up the way to control dynamical behaviour of molecular systems. With these developments, the long held dream of a chemist to manipulate chemical reactions, for example to generate desired chemical products with high efficiency, while at the same time reducing unwanted side products, is progressively becoming true. In the last two decades, the control of molecular dynamics via shaped laser pulses has experienced considerable progress from a theoretical concept ${ }^{3-6}$ to increasing number of successful experiments. ${ }^{7-10}$ With the hope of quantum computing becoming a reality in years to come; coherent preparation of molecular states has gained a lot of importance. ${ }^{11}$

Ever since their invention, lasers have been viewed as an ideal tool for controlling chemical processes because of their monochromatic nature. Growth in laser technology has helped us to understand that the dynamics of a strongly coupled light-matter system can be influenced by alteration of temporal, spectral

*For correspondence and phase distributions of the radiation coupled to the system. Several methods of controlling molecular dynamics, like the coherent control ${ }^{12-15}$ and optimal control $^{16-18}$ using laser fields have recently gained popularity. While the former uses quantum mechanical properties of interference of dynamical paths to regulate the production of a state, the latter provides a more general prescription using calculus of variations.

Recently, a large number of researchers have focused greater attention on optimal control of quantum states, leading to an extensive theoretical and numerical work in this area. Zhu et $a l^{19}$ have presented a family of new iteration methods to solve quantum optimal control problems. They have demonstrated the efficiency of their algorithm on a typical one-dimensional system consisting of the excitation from the ground state to the first excited state in a Morse potential of the $\mathrm{O}-\mathrm{H}$ bond. Balint-Kurti et $a l^{20,21}$ have illustrated the utilization of polarization forces for optimal control of the vibrational excitation of the homonuclear diatomic molecule $\left(\mathrm{H}_{2}\right)$, using the conjugate gradient method for optimization.

In this work we apply the conjugate gradient method for the optimal control of molecular vibrational excitations in the heteronuclear diatomic molecule, HF. We obtain optimal fields of different pulse durations for population transfer from an initial vibrational state to the desired target vibrational state of the hydrogen fluoride molecule. We have treatęg 
the hydrogen fluoride molecule. We have treated the interaction of the molecule with the laser light within dipole approximation, i.e. it consists only of the interaction of the electric field vector of the radiation with the geometry-dependent electric dipole moment of the molecule. ${ }^{22,23} \mathrm{We}$ have carried out calculations using Morse potential as well as a potential energy function obtained using $a b$ initio quantum mechanical electronic structure calculations; and compared the results. Two pulse durations have been considered: (a) 30,000 a.u. and (b) 60,000 a.u. respectively, for the following two vibrational excitation processes, where the first one is a fundamental excitation from the lowest to the next vibrational energy level and the second is an excitation process starting from a higher vibrational level.

$$
\begin{aligned}
& \operatorname{HF}(v=0) \rightarrow \operatorname{HF}(v=1), \\
& \operatorname{HF}(v=3) \rightarrow \operatorname{HF}(v=4) .
\end{aligned}
$$

The conjugate gradient method for optimization used in this work is described in $\S 2$. The results are discussed in $\S 3$. Finally, we summarise the conclusions in $\S 4$.

\section{Theoretical and computational methods}

\subsection{Theory}

Our aim is to design a laser pulse for population transfer from an initial state to a desired dynamical goal at a fixed time while minimizing the pulse energy. The time evolution of the quantum mechanical state $\psi(t)$ under the influence of an external field $\varepsilon(t)$, within the dipole approximation, is governed by the time-dependent Schrödinger wave equation

$$
i \hbar \frac{\partial}{\partial t}|\psi(t)\rangle=\left[\hat{H}_{0}-\hat{\mu} \varepsilon(t)\right]|\psi(t)\rangle,
$$

where $\hat{H}_{0}$ and $\hat{\mu}$ are the system Hamiltonian and the dipole moment operator, respectively.

For this purpose we construct a cost functional, $J[\varepsilon]$, which depends on the optimal driving field. The cost functional contains mainly three terms,

$$
J[\varepsilon]=J_{o}+J_{p}+J_{c},
$$

where, $J_{o}$ contains the physical objective i.e. $J_{o}=$ $\left|\left\langle\psi_{i}(T) \mid \psi_{f}(T)\right\rangle\right|^{2}, J_{p}$ contains the penalties, i.e.

$$
J_{p}=-\int_{0}^{T}[\varepsilon(t)]^{2} \mathrm{~d} t,
$$

and $J_{c}$ the dynamical constraint, that is the Schrödinger equation is obeyed at every point of time,

$$
i \eta \frac{\partial\left|\Psi_{i}(t)\right\rangle}{\partial t}=\hat{H}\left|\psi_{i}(t)\right\rangle .
$$

The Grand functional to be optimized takes the following shapes:

$$
\begin{array}{r}
J[\varepsilon(t)]=\left|\left\langle\psi_{i}(T) \mid \phi_{f}(T)\right\rangle\right|^{2}-\alpha_{0} \int_{0}^{T}[\varepsilon(t)]^{2} \mathrm{~d} t-2 \Re e \\
{\left[\left\langle\psi_{i}(T) \mid \phi_{f}(T)\right\rangle \int_{0}^{T}\left\langle\chi_{f}(t)\left|\frac{\partial}{\partial t}+i \hat{H}\right| \psi_{i}(t)\right\rangle \mathrm{d} t\right]}
\end{array}
$$

The function $\psi_{i}(t)$ is the initial wave function propagated to time $T$ by the optimal laser field $\varepsilon(t)$ and $\phi_{f}(T)$ is the target vibrational state specified at the final time $T$. The function $\chi_{f}(t)$ can be regarded as a Lagrange multiplier introduced to assure satisfaction of the Schrödinger equation. The factor $\alpha_{0}$ is a positive weighting parameter that specifies the weight of the laser radiation energy to the functional. Varying the grand functional with respect to the initial wave function $\psi_{i}(t)$, the Lagrange multiplier $\chi_{f}(t)$ and the electric field $\varepsilon(t)$ leads to the following set of equations:

$$
\begin{aligned}
& i \frac{\partial \psi_{i}(t)}{\partial t}=\hat{H} \psi_{i}(t), \psi_{i}(0)=\phi_{i}(0), \\
& i \frac{\partial \chi_{f}(t)}{\partial t}=\hat{H} \chi_{f}(t), \chi_{f}(T)=\phi_{f}(T), \\
& \alpha_{0} \varepsilon(t)=-\Im m\left(\left\langle\chi_{f}(t)|\mu| \psi_{i}(t)\right\rangle\left\langle\psi_{i}(T) \mid \phi_{f}(T)\right\rangle\right),
\end{aligned}
$$

These coupled differential equations can be solved iteratively. In order to maximize our grand functional (4), subject to the constraints in (5) and (6) we have used the conjugate-gradient method discussed below.

2.1b Conjugate gradient method: In order to theoretically design a laser field that can selectively excite HF from its initial vibrational state to specific vibrational excited state, calculation is carried out 
using the conjugate gradient method ${ }^{21,24}$ by maximizing the value of the grand functional $J[\varepsilon(t)]$ mentioned in (4). The gradient of $J[\varepsilon(t)]$ including the penalty term

$$
J_{p}=-\int_{0}^{T}[\varepsilon(t)]^{2} \mathrm{~d} t
$$

and the dynamical constraint of Schrödinger equation with respect to the variation of $\varepsilon$ at time $t$ is given by

$$
\begin{aligned}
g^{k}(t) \equiv & \frac{\partial J^{k}}{\partial \varepsilon^{k}(t)}=-2 s(t)\left[\alpha_{0}\left[\varepsilon^{k}(t)\right]+\right. \\
& \left.\Im \operatorname{Im}\left\langle\chi_{f}(t)|\mu| \psi_{i}(t)\right\rangle\left\langle\psi_{i}(T) \mid \phi_{f}(T)\right\rangle\right],
\end{aligned}
$$

where the superscript $k$ indicates the iteration number in the optimization cycle. The factor $s(t)$, is a Gaussian envelope function is given by

$$
s(t)=e^{-(t-T / 2)^{2} /(T / 6)^{2}},
$$

Several groups have used $s(t)=\sin ^{2}(\pi t / T)$ as envelope function. Results using such a sinusoidal factor are communicated by our own group elsewhere. ${ }^{25}$

The initial wave function, $\psi_{i}(t)$, and the Lagrange multiplier, $\chi_{f}(t)$, are propagated in time using the second order split-operator method. ${ }^{26,27}$ The PolakRibiere-Polyak ${ }^{28}$ search direction can be calculated using the (8) as

$$
d^{k}\left(t_{i}\right)=g^{k}\left(t_{i}\right)+\lambda^{k} d^{k-1}\left(t_{i}\right)
$$

where

$$
\lambda^{k}=\frac{\sum_{i} g^{k}\left(t_{i}\right)^{T}\left(g^{k}\left(t_{i}\right)-g^{k-1}\left(t_{i}\right)\right)}{\sum_{i} g^{k-1}\left(t_{i}\right)^{T} g^{k-1}\left(t_{i}\right)},
$$

$k=2,3, \ldots$ and $d^{1}\left(t_{i}\right)=g^{1}\left(t_{i}\right)$. The function $\lambda^{k}$ is the conjugate gradient parameter and the summation is over all time intervals. A line search is then performed along this direction to determine the maximum value of the objective functional. To prevent the algorithm from sampling $\varepsilon(t)$ values outside of a defined field amplitude range $\left[\varepsilon_{\min }, \varepsilon_{\max }\right]$ during the line search, the direction is manipulated appropriately. ${ }^{29}$
The projected search direction is Fourier transformed to obtain a function of frequency, and in order to restrict the frequency components of the electric field within a specified range 20th-order Butterworth band pass filter ${ }^{30,31}$ is used. The field is updated using the following expression as

$$
\varepsilon^{k+1}(t)=\varepsilon^{k}(t)+\lambda d_{P}^{k}(t) s(t),
$$

where $\lambda$ is determined by the line search and $d_{P}^{k}(t)$ is a projected direction.

\subsection{Ab initio molecular electronic structure method}

We modelled the atomic interactions using the Morse potential as well as calculated it using quantum mechanical calculations (figure 1 ). The $a b$ initio calculations were performed using MP2/6-31G (d, p) basis set. Gaussian $03^{32}$ suite of programs was used for all $a b$ initio molecular electronic structure calculations carried out in this work. It can be seen from figure 1 that neither of the potential energy curves is accurate at internuclear distances much smaller than the equilibrium value. The $a b$ initio curve is shallower and also less harmonic.

\section{Results}

We have chosen a heteronuclear diatomic system for studying the vibrational excitations from an initial

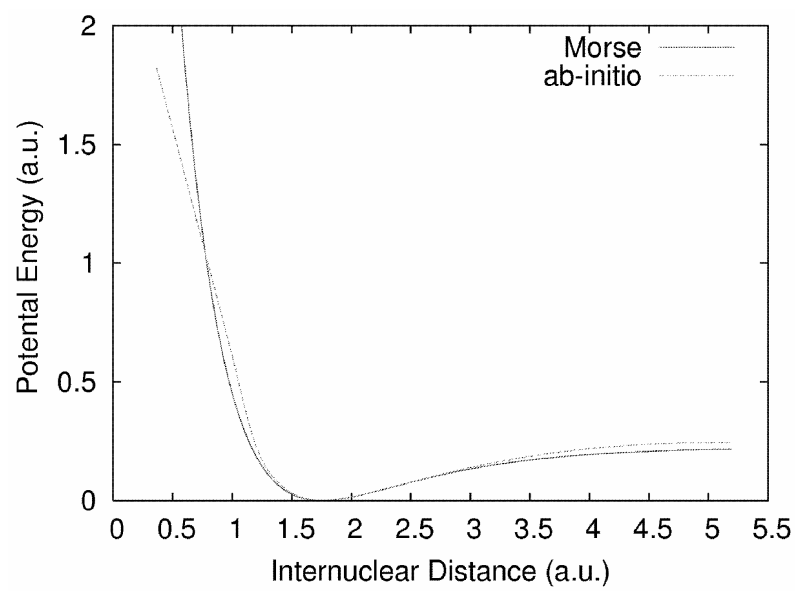

Figure 1. Potential Energy curves for HF: Morse potential model (eq. (12); -) and calculated $a b$ initio [using MP2/6-31G $(d, p)$ basis set] $(\cdots)$. 
Table 1. Comparison of transition probabilities and grand functionals for different pulse durations for transitions in HF using a Morse potential model.

\begin{tabular}{lccccc}
\hline \multirow{2}{*}{ Transition } & \multicolumn{2}{c}{$P$} & \multicolumn{2}{c}{$J$} \\
\cline { 2 - 3 } \cline { 5 - 6 } & $T=30,000$ a.u. & $T=60,000$ a.u. & $T=30,000$ a.u. & $T=60,000$ a.u. \\
\hline $0 \rightarrow 1$ & 0.98605 & 0.99715 & & 0.82966 & 0.92018 \\
$3 \rightarrow 4$ & 0.99821 & 0.99965 & & 0.94018 & 0.97239 \\
\hline
\end{tabular}
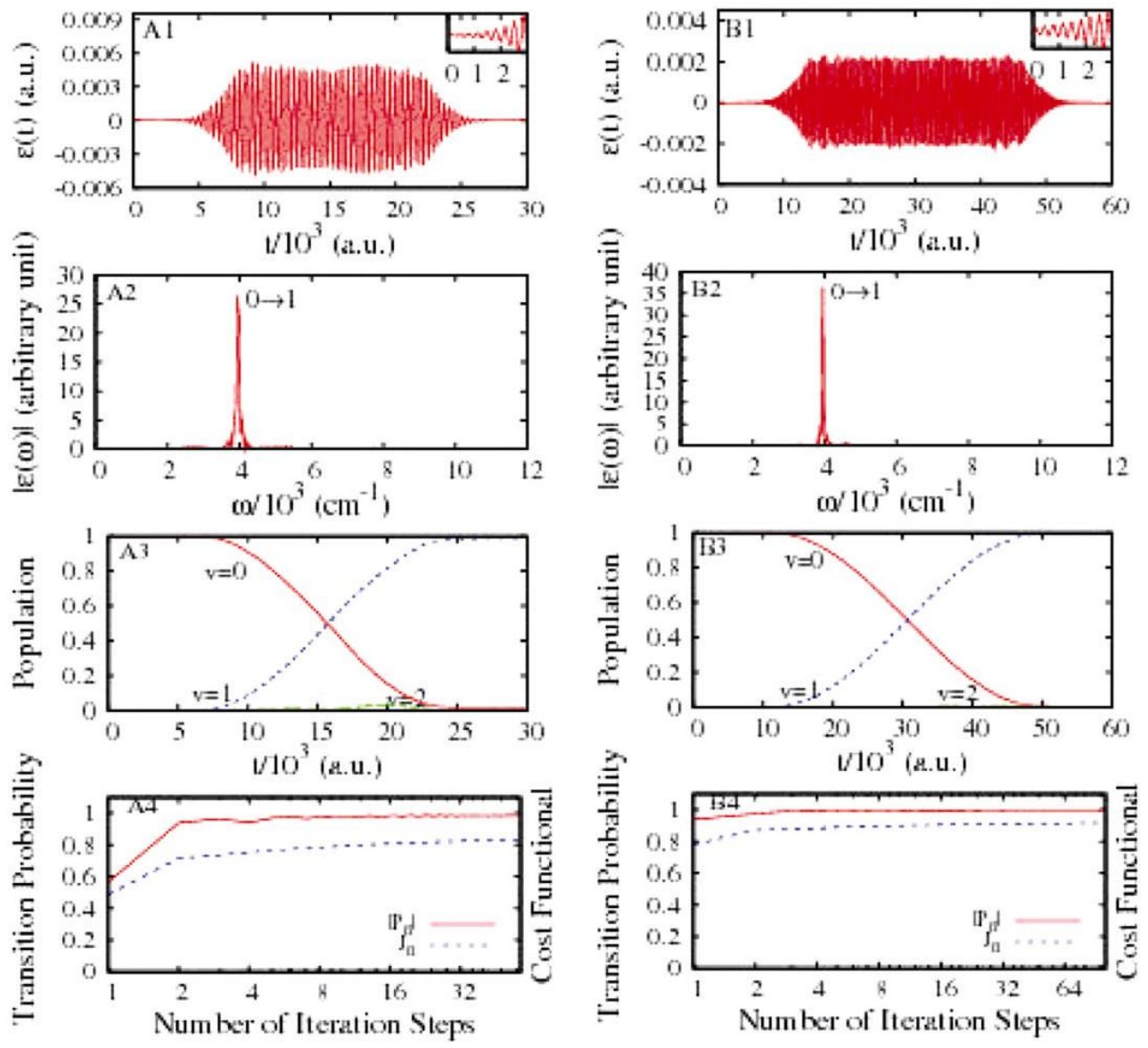

Figure 2. Optimized field as a function of time (A1, B1), frequency spectrum of the optimized field (A2, B2), population analysis of relevant states (A3, B3), convergence of transition probability and grand functional (A4, B4) for $v=0 \rightarrow v=1$ transition in HF using a Morse potential model for different pulse durations, 30,000 a.u. (A1-A4) and 60,000 a.u. (B1-B4).

vibrational state to a target vibrational state in a Morse potential of the HF molecule in the prescribed time interval $\mathrm{T}$. Our objective is to control the population transfer from an initial state to a target state while minimizing the fluence. The Fourier grid Hamiltonian method $^{33}$ is used to compute the vibrational energies and eigenfunctions of the initial and target vibrational states. The atomic interaction is modelled using Morse potential

$$
V(x)=D_{e}\left[1 \cdot 0-e^{-\beta\left(x-x_{e}\right)}\right]^{2},
$$

where the parameters $D_{e}=0.2250073497, x_{e}=$ 1.7329 , and $\beta=1.1741$ in a.u., and the dipole moment used is given by ${ }^{34}$ 

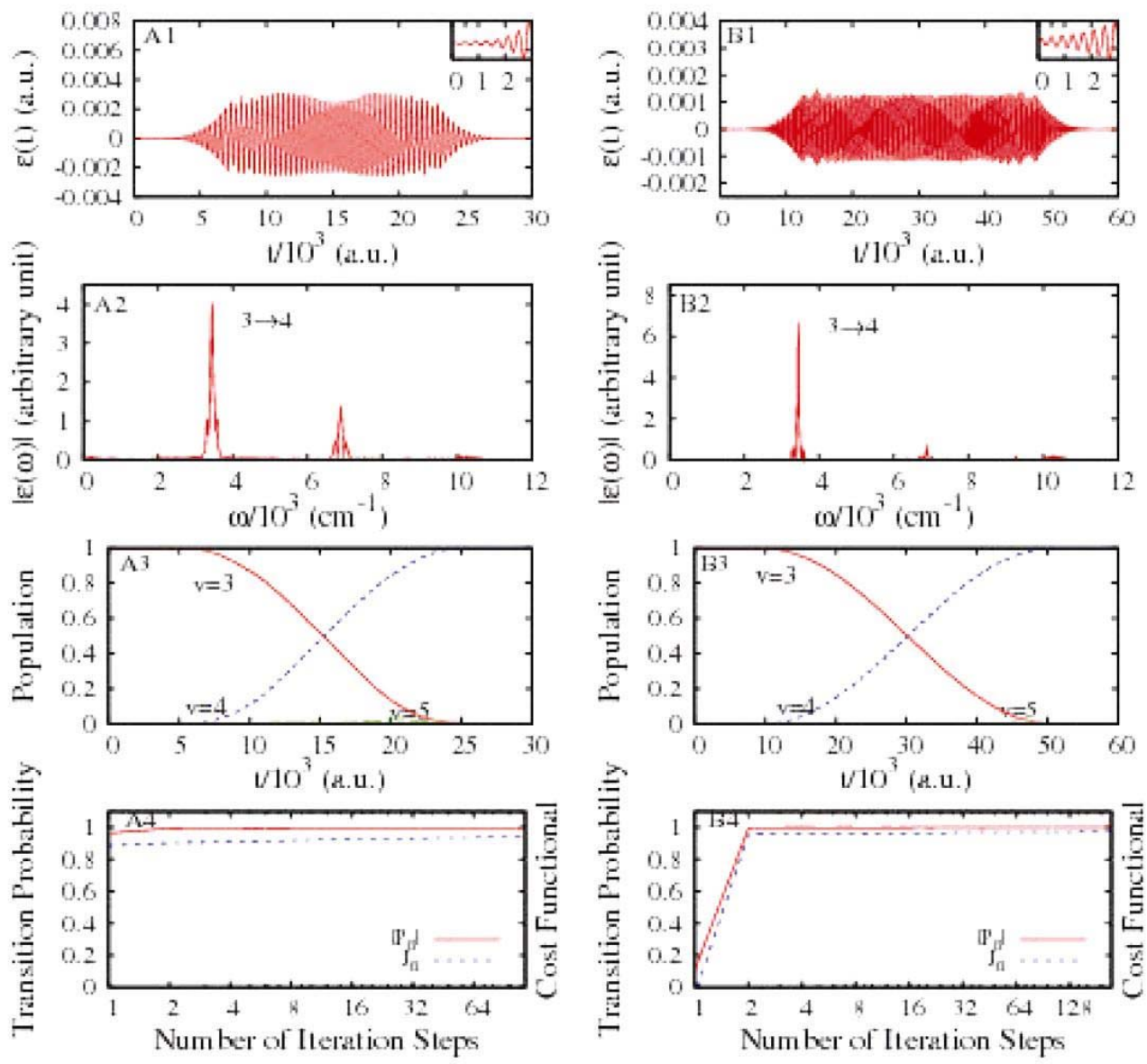

Figure 3. Optimized field as a function of time (A1, B1), frequency spectrum of the optimized field (A2, B2), population analysis of relevant states (A3, B3), convergence of transition probability and grand functional (A4, B4) for $v=3 \rightarrow v=4$ transition in HF using a Morse potential model for different pulse durations, 30,000 a.u. (A1-A4) and 60,000 a.u. (B1-B4).

$$
\mu(x)=\mu_{0} x e^{-\sigma x^{4}}
$$

where $\mu_{0}=0.4535$ and $\sigma=0.0064$ in a.u.

We have carried out calculations for the transitions $v=0 \rightarrow v=1$ and $v=3 \rightarrow v=4$ using conjugate gradient method for pulse durations of 30,000 a.u. and 60,000 a.u. and compared the results with those obtained from the $a b$ initio electronic structure calculations. The pulse length is discretized into 8191 points. The maximum electric field strength for all computed optimized laser pulses is limited to 0.005 a.u. with a fluence weight of $\alpha_{0}=1$.

\subsection{Results obtained using Morse potential as a model for atomic interaction}

(a) Excitation: $v=0 \rightarrow v=1$ : Results of the optimization process and the action of the theoretically de- signed laser pulses using optimal control theory, for the two transitions described in (1) are summarized in table 1. For all the transitions the initial trial field was chosen to have the following form:

$$
\varepsilon_{i}(t)=0.005 \sin \left(\omega_{i f} t\right) s(t)
$$

where $\omega_{i f}=\left(\omega_{f}-\omega_{i}\right)$ corresponds to the transition frequency from initial to the final vibrational state of HF molecule. For the first transition, the converged optimized electric fields for the duration 30,000 a.u. and 60,000 a.u. are shown in plots A1, B1 of figure 2. Plots $\mathrm{A} 2$ and $\mathrm{B} 2$ present the frequency analysis of the optimized fields displaying a sharp peak at $3958 \mathrm{~cm}^{-1}$ which corresponds to the resonance frequency for the transition from ground vibrational state to the first excited vibrational state of $\mathrm{HF}$ molecule. The plots A3, B3 in figure 2 show the 
population of the ground and excited vibrational states as a function of time over the chosen duration of the laser pulses. As expected, as the time increases, population of the ground vibrational state decreases while that of target vibrational state increases. The plots A4, B4 in figure 2 show the convergence behaviour of the optimized probability as well as objective functional with the number of iterative steps involved. After few iterative steps, objective functional converges to 0.82966 for the shorter pulse $(T=30,000$ a.u. $)$ and to 0.92018 for the longer pulse ( $T=30,000$ a.u) corresponding to a population transfer in each case of nearly $100 \%$.

(b) Excitation: $v=3 \rightarrow v=4$ : While for the simplest case of excitation discussed above, a state to state transition using the fundamental resonance frequency seems to be the obvious and dominant dynamics, greater complexity is expected as we go to higher states. We thus examine the possibility of designing a laser field for exciting the system from a higher vibrational state $(v=3)$ to the next vibrational state $(v=4)$. The converged optimized electric fields and their frequency distribution are shown in plots (A1, A2) and (B1, B2) of figure 3 respectively. Plots $\mathrm{A} 2$ and $\mathrm{B} 2$ in the same figure show a dominant peak corresponding to the vibrational transition from $v=3 \rightarrow v=4$ at $3416 \mathrm{~cm}^{-1}$ and a small contribution from the 2 nd harmonic. The width in the peaks indicates marginal involvement of other states with nearly the same transition energy. Plots A3 and B3 show a complete transfer of population from initial state to
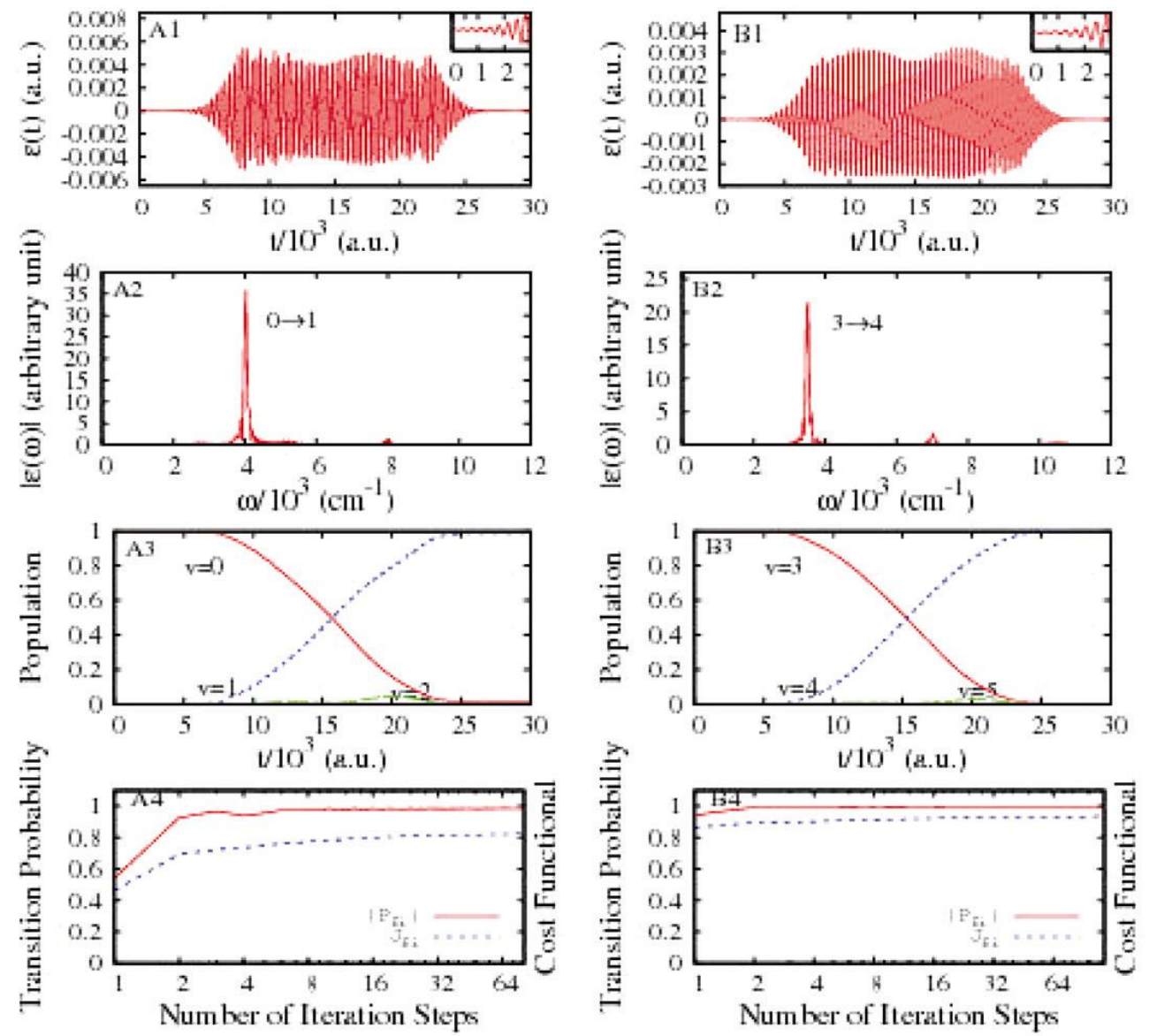

Figure 4. Optimized field as a function of time (A1, B1), frequency spectrum of the optimized field (A2, B2), population analysis of relevant states (A3, B3), convergence of transition probability and grand functional (A4, B4) for $v=0 \rightarrow v=1$ and $v=3 \rightarrow v=4$ transitions for pulse durations 30,000 a.u for HF potential energy curve calculated using $a b$ initio quantum mechanical method using MP2/6-31G $(d, p)$ basis set. 
Table 2. Comparison of transition probabilities $(P)$ and cost functional $(J)$ for $v=0 \rightarrow v=1$ and $v=3 \rightarrow v=4$ transitions for pulses of duration 30,000 a.u.

\begin{tabular}{|c|c|c|c|c|c|c|}
\hline \multirow[b]{2}{*}{ Transition } & \multicolumn{2}{|c|}{ Energy $\left(\mathrm{cm}^{-1}\right)$} & \multicolumn{2}{|c|}{ Morse potential } & \multicolumn{2}{|c|}{$a b$ initio electronic calculation } \\
\hline & Morse & $a b$ initio & $P$ & $J$ & $P$ & $J$ \\
\hline $0 \rightarrow 1$ & 3958 & 4029 & 0.98605 & 0.82966 & 0.98709 & 0.82424 \\
\hline $3 \rightarrow 4$ & 3445 & 3512 & 0.99821 & 0.94018 & 0.99786 & 0.93558 \\
\hline
\end{tabular}

final state over the duration of pulse. The convergence behaviour of objective functional and optimized probability versus number of iterative steps is shown in plots A4, B4 of figure 3 .

On comparing the results in table 1 , it is seen that with the increase in pulse duration from 30,000 a.u. to 60,000 a.u., final transition probabilities and value of final cost functional increases. This increase occurs simultaneous with a decrease in peak amplitude of the field, thus reducing the total fluence in the expression for the cost functional, (4). The increase in the value of cost functional as we go from the transition between the lower states $v=0 \rightarrow v=1$, to that between the higher states, $v=3 \rightarrow v=4$, occurs primarily because of decrease in energy gap, thus reducing the fluence of the field required. Interestingly, this increase may also be due to constructive interference between alternate dynamical paths involving similar energies available for the $v=3 \rightarrow$ $v=4$ transition.

\subsection{Results obtained using ab initio calculations}

We modelled the atomic interactions by the potential obtained using $a b$ initio calculations also. Table 2 summarizes the comparison of the results obtained using Morse potential and electronic potential energy obtained using $a b$ initio for the transitions $v=0 \rightarrow$ $v=1$ and $v=3 \rightarrow v=4$ for a single pulse duration of 30,000 a.u. Plots A1, B1 of figure 4 show the converged optimized fields for both the vibrational transitions for pulse durations 30,000 a.u. using $a b$ initio electronic structure calculation. The corresponding power spectra and frequency distribution are shown in plots A2, B2 of figure 4. From plots $\mathrm{A} 3, \mathrm{~B} 3$ in the same figure, we can see how the populations of the initial state and target state vary with time over the duration of pulse. The convergence behaviours of objective functional and optimized probability versus number of iterative steps are shown in plots A4, B4 of figure 4.
On comparing these results we find that the values of transition probabilities are nearly the same, about $99 \%$ for both the potentials. The values of cost functional are also nearly the same for fixed pulse duration and it increases with pulse duration for reasons explained earlier. The insignificant difference in results using the two potential energy curves reflects the fact that for dynamics near the equilibrium internuclear distance the difference between the two potentials is negligible. For even higher excitations, as the two potential energy curves begin differing from each other, we are likely to see greater differences in results of OCT calculations.

\section{Conclusions}

The optimization procedure described above gives reliable fields for the desired dynamical goals. We have applied the method to different vibrational excitations for heteronuclear molecule (HF) and obtained the optimal fields which will achieve the desired dynamical goal. Since the dynamics for the chosen potentials are confined to distances not very far from the equilibrium value o the internuclear distance, the converged values of the cost functional are close to each other for fixed duration of the laser pulse. The values of transition probability are almost the same for both the potentials. Application of the method to more complex problems is in progress. Issues including robustness of the optimal result to environmental noise are also being investigated.

\section{Acknowledgement}

We thank Prof Gabriel Balint-Kurti, School of Chemistry, University of Bristol, for valuable discussions. H S thanks Department of Science and Technology, Government of India for support during earlier stages of the work, and United Kingdom India Education Research Initiative (UKIERI) and British Council for supporting a visit to the Balint- 
Kurti group. SS and PS thank Council of Scientific and Industrial Research (CSIR). Government of India for research fellowships.

\section{References}

1. Rice S and Zhao M 2000 Optical control of ,olecular dynamics (John Wiley Sons: USA)

2. Shapiro M and Brumer P 2003 Principles of the quantum control of molecular Processes (John Wiley Sons: USA)

3. Tannor D J and Rice S 1985 J. Chem. Phys. 835013

4. Brumer P and Shapiro M 1986 Chem. Phys. Lett. 126 541

5. Shi S and Rabitz H 1990 J. Chem. Phys. 92364

6. Gross P, Singh H, Rabitz H, Mease K and Huang G M 1993 Phys. Rev. A47 4593

7. Assion A, Baumert T, Bergt M, Brixner T, Kiefer B, Seyfried V, Strehle M and Gerber G 1998 Science 282919

8. Levis R J, Mnkir G M and Rabitz H 2001 Science 292709

9. Vajda S et al 2001 Chem. Phys. 267231

10. Nuernberger $P$, Vogt $G$, Brixner $T$ and Gerber $G$ 2007 Phys. Chem. Chem. Phys. 92470

11. Tesch C M and Vivie-Riedle R de 2002 Phys. Rev. Lett. 89157901

12. Shapiro M and Brumer P 1989 Acc. Chem. Res. 22407

13. Brumer P and Shapiro M 1992 Ann. Rev. Phys. Chem. 43257

14. Bergmann K, Theuer H and Shore B W 1998 Rev. Mod. Phys. 701003

15. Goswami D 2003 Phys. Rep. 374385

16. Peirce A P, Dahleh M A and Rabitz H 1988 Phys. Rev. A37 4950

17. Dahleh M A, Peirce A P and Rabitz H 1990 Phys. Rev. A42 1065
18. Palao J P and Kosloff R 2003 Phys. Rev. A68 062308

19. Zhu W, Botina J and Rabitz H 1998 J. Chem. Phys. 1081953

20. Balint-Kurti G G, Zou S and Brown A 2007 Adv. Chem. Phys. (in press)

21. (a) Balint-Kurti G G, Manby F R, Ren Q, Artamonov M, Ho T and Rabitz H 2005 J. Chem. Phys. 122 084110 (b) Ren Q, Balint-Kurti G G, Manby F R, Artamonov M, Ho T and Rabitz H 2006 J. Chem. Phys 124014111

22. Sundermann K and Vivie-Riedle R de 1999 J. Chem. Phys. 1101896

23. Shah S P and Rice S A 2000 J. Chem. Phys. 1136536

24. Press W H, Teukolsky S A, Vetterling W $\mathrm{T}$ and Flannery B P 2000 Numerical Recipes in FORTRAN, (Cambridge U. P.)

25. Kumar $P$, Sharma $S$ and Singh $H$ (in this issue)

26. Feit M D and Fleck Jr J A 1983 J. Chem. Phys. 78 301

27. Feit M D and Fleck Jr J A 1984 J. Chem. Phys. 80 2578

28. Polak E 1971 Computational methods in optimization, mathematics in science and engineering (New York: Academic), vol. 77

29. Birgin E G, Martinez J M and Raydan M 2000 SIAM J. Optim. 101996

30. Digital Signal Processing (eds) L R Rabiner and C M Rader 1972 (New York: IEEE)

31. Gross P, Neuhauser D and Rabitz H 1992 J. Chem. Phys. 962834

32. (a) Becke A D 1993 J. Chem. Phys. 985648 (b) Lee C, Yang W, Parr R G 1988 Phys. Rev. B37 785 (c) Frisch M J et al 2004 Gaussian 03, revision C.02, Gaussian Inc. Wallingford CT

33. Clay C Marston and Balint-kurti G G 1989 J. Chem. Phys. 913571

34. Stine J R and Noid D W 1979 Opt. Commun. 31161 